\title{
Hybrid Organic-Inorganic Silica Gel Carriers with Controlled Drug-Delivery Properties
}

\author{
Laura Contessotto, ${ }^{[\mathrm{a}]}$ Elena Ghedini ${ }^{[\mathrm{a}]}$ Francesco Pinna, ${ }^{[\mathrm{a}]}$ Michela Signoretto, ${ }^{*[a]}$ \\ Giuseppina Cerrato, ${ }^{[b]}$ and Valentina Crocella ${ }^{[b]}$
}

\begin{abstract}
Pure and modified silica materials were synthesised by a sol-gel process and used as carrier for the controlled release of ibuprofen, selected as model drug. A one-step synthesis was optimised for the preparation of various silica-drug composites by using tetraethoxysilane and 3-aminopropyltriethoxysilane as precursors at different molar ratios. The presence of aminopropyl groups on the silica surface influences the drug-delivery rate leading to a high degree the desorption process controlled.
\end{abstract}

Keywords: drug delivery $\cdot$ ibuprofen $\cdot$ organic-inorganic hybrid composites • silica materials • sol-gel processes

\section{Introduction}

Controlled drug delivery occurs when a matrix, usually a polymer, whether natural or synthetic, is opportunely combined with a drug or other active agents in such a way that the active agent is released from the material in a controlled manner. The goal of many of the original controlled-release systems was to achieve a delivery profile that would yield a high blood level of the drug over a long period of time. The key point with traditional drug administration is that the blood level of the agent should remain between a maximum value, which may represent a toxic level, and a minimum value, below which the drug is no longer effective. In controlled drug-delivery systems designed for long-term administration, the drug level in the blood should remain constant within the desired range for an extended period of time. Controlled-delivery systems offer other advantages, for example, the need for fewer administrations, optimal use of the specific drug, and increased patient compliance. These

[a] Dr. L. Contessotto, E. Ghedini, Prof. F. Pinna, M. Signoretto Department of Chemistry University Cà Foscari Consortium INSTM-RU of Venice

Calle Larga Santa Marta, 2137, 30123 Venice (Italy) Fax: (+39) 041-2348517

E-mail:miky@unive.it

[b] Dr. G. Cerrato, Dr. V. Crocellà

Department of Chemistry IFM-NIS Centre of Excellence University of Turin and Consortium INSTM-RU of Turin via P. Giuria, 10125 Turin (Italy) advantages can be all significant, but the potential disadvantages cannot be ignored: the possible toxicity or non-biocompatibility of the materials used, undesirable byproducts of degradation, any surgery required to implant or remove the system, the chance of patient discomfort from the delivery device and the higher cost of controlled-release systems compared with traditional pharmaceutical formulations. The ideal drug-delivery system should be inert, biocompatible, mechanically strong, comfortable for the patient, capable of achieving high drug loading, safe from accidental release, simple to administer and remove and easy to fabricate. Some of the drug carriers currently in use include soluble polymers, microparticles made of insoluble or biodegradable natural and synthetic polymers, microcapsules, cells, lipoproteins, liposomes and micelles. Recently silica matrices have been investigated for encapsulation of biomolecules such as enzymes, antibodies and cells. ${ }^{[1,2]}$ Silica matrices show high biocompatibility-biodegrability ${ }^{[3-5]}$ and resistance to microbial attack and they exhibit higher mechanical strength, enhanced thermal stability and negligible swelling in organic solvents, compared to most organic polymers. All these features make silica supports promising carriers for the drugdelivery systems formulation. Several research groups have investigated both drug storage and delivery properties of ordered mesoporous silica ${ }^{[6-10]}$ and of sol-gel silica materials. ${ }^{[11-13]}$ A major advantage in the use of a sol-gel is that the matrix can be generated in the presence of the drug molecule in a one-step process; moreover, the physicochemical features of silica could be suitably modulated by the control and the opportune variation of the parameter process (composition of precursor mixture, catalysts, $\mathrm{pH}$, ageing). Re- 
cently we have examined the delivery behaviour of ibuprofen from hybrid inorganic-organic silica carriers. ${ }^{[14,15]}$ In particular we have obtained promising results from silica matrices modified with methyl groups prepared by a sol-gel approach. With the addition of these organic functions, we have decreased the amount of surface silanol groups, leading to a more hydrophobic surface that allows an effective control of the ibuprofen release: in fact, in this case, it is very close of that expected for an ideal drug-delivery system (slow and gradual). Unfortunately with these matrices we have obtained only a partial release $(70 \mathrm{wt} \%)$ of the total drug initially loaded. It is, instead, desirable, to achieve a gradual drug release and at the same time characterised by a highest amount of the delivered drug. In the present study, the silica structure is modified with the introduction of hydrophilic groups by using the (3-aminopropyl) triethoxysilane (APTES) as precursor in a sol-gel process. APTES is employed in many fields of the modern chemistry and technology for chemical modification of various oxide surfaces and for surface coatings $;{ }^{[16]}$ moreover, in this last decade several reports have been published on its use as modifier agent of porous silica employed as carriers for encapsulation and delivery of pharmaceutical drugs. ${ }^{[1,17]}$ However, to the best of our knowledge, only a few studies ${ }^{[18]}$ have been reported, up to now, on hybrid amine-silica carriers prepared by sol-gel methods used for the sustained release of drugs. The modification with APTES changes dramatically the physicochemical properties of the silica matrix affecting, in particular, both reactivity and stability of the obtained materials in an aqueous medium. This aspect is very important for a drug-delivery system in which the matrix degradation can affect to a high degree the sustained release. Moreover, the aminopropyl groups can increase the strength of interaction between the functional groups and the drug molecule if opportunely selected. It is worth noting that the hydrolysis products of APTES elicit a sensitisation response neither from at high dosage nor after long period of exposition and administration: in fact, both in vivo and in vitro screening assays have not revealed any evidence of genotoxic potential. $^{[19]}$ This consideration is very important in view of the final application of the carrier here under investigation. The goal of this work is the optimisation of a one-step sol-gel process for the synthesis of hybrid amine-silica carriers for the controlled release of ibuprofen. We have, in particular, studied the optimal composition (amount of the functional groups) of the final material, in order to obtain a gradual and controlled release, but, at the same time, leading to a high final amount of drug released.

\section{Results and Discussion}

$100 \%$ Tetraethoxysilane (TEOS) silica matrix: All silica carriers studied in this work have been prepared by a one-step synthesis following the sol-gel method. This low-temperature process offers several advantages: in particular, it assures high homogeneity of the final products, ${ }^{[13]}$ with the possibility of various formation processes that allow the strict control of the physicochemical properties of the final systems. In the present work we have used ibuprofen with tetraethoxysilane (TEOS) as silica source, following the procedure reported in previous work. ${ }^{[15]}$ We have obtained a monolithic silica/ibuprofen gel (Figure 1): it was limpid,

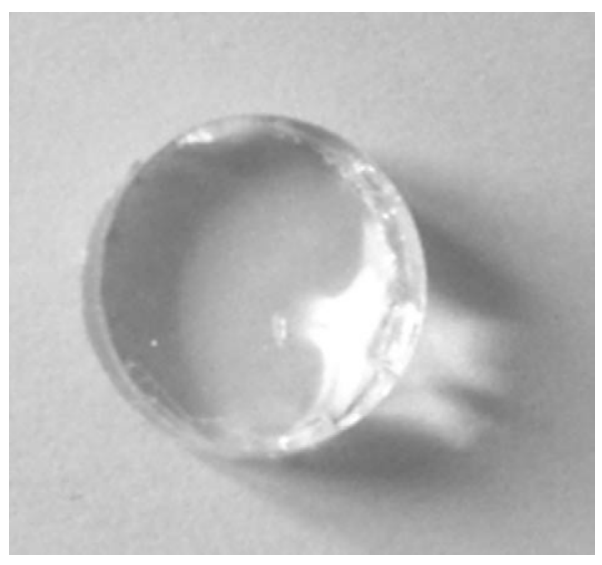

Figure 1. Picture of a silica/ibuprofen tablet

transparent and homogeneous, and was used as reference frame. The amount of drug released from TEOS-derived samples (expressed as \% of ibuprofen on the total amount of ibuprofen present) is shown as a function time in Figure 2. The data were collected from a gel aged for ten days, the time necessary to obtain a stable carrier. ${ }^{[15]}$ The delivery profile exhibits a pronounced initial release of $50 \%$ of ibuprofen under $30 \mathrm{~min}$ followed by a slightly slower release rate. A kinetic of this type is not proper for a controlled delivery system that should gradually release drugs

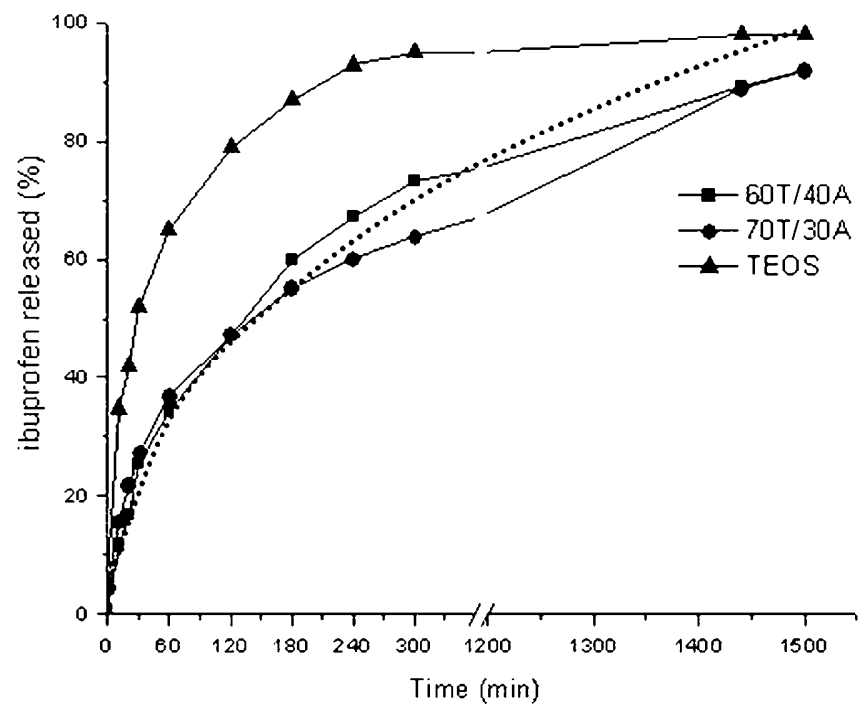

Figure 2. Ibuprofen delivery from $100 \%$ TEOS carrier and from modified samples. Comparison of the experimental release with the profile assumed for an ideal drug-delivery system (dashed line). 
at a constant rate, thereby maintaining (in vivo) drug concentration within the therapeutic range and thus eliminating the need for frequent re-dosages. However, this is not surprising: in fact, drug release is generally known to be relatively fast in TEOS-based xerogel without dopant. ${ }^{[4]}$ The liberation process of a drug from a solid carrier is essentially governed by both matrix dissolution and diffusion, ${ }^{[3]}$ which are strictly connected with the physicochemical properties of the matrix itself. In particular, both hydrophilic/hydrophobic character of the matrix and the nature of interaction between the embedded molecule and the surface of the carrier play a key role. In the case of the TEOS-derived silica matrix, ibuprofen can interact with the few surface silanol groups only by weak interactions (mainly by hydrogen bonding) and this could probably explain its fast release kinetic (Scheme 1). We checked if this speculation could be

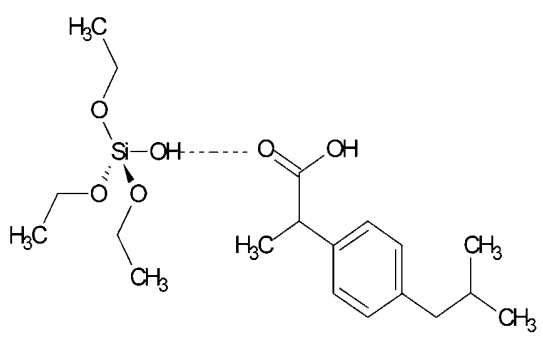

Scheme 1. Example of interaction between ibuprofen and the silanol groups on the silica surface

supported by any experimental evidence: for this reason, FTIR spectroscopy of the powders themselves has been resorted to in order to investigate the nature of the TEOS/ibuprofen interaction. The $100 \%$ TEOS matrix exhibits the known spectroscopic features (see curve 1 in Figure 3), typical of a plain silica-based system. ${ }^{[20]}$ In particular, in the

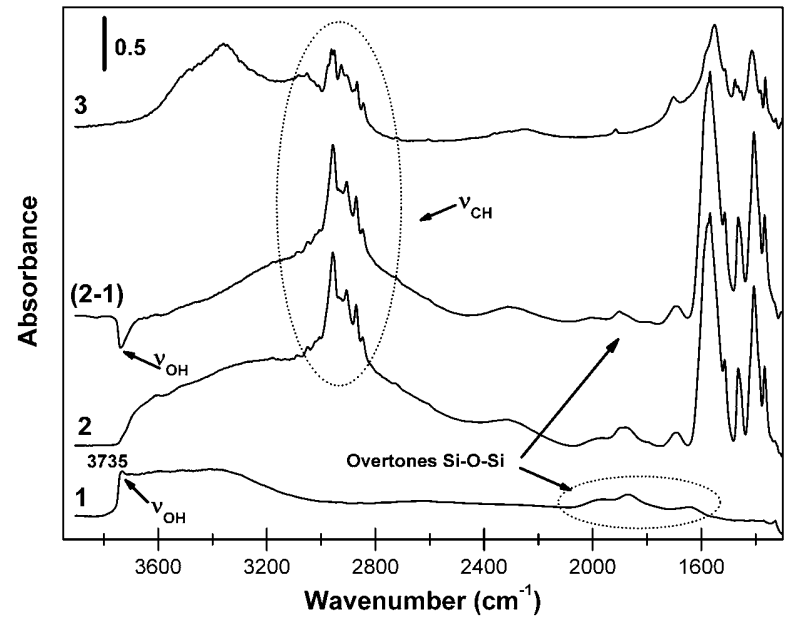

Figure 3. Absorbance FTIR spectra in the $3950-1300 \mathrm{~cm}^{-1}$ range relative to several samples outgassed at beam temperature for one hour: curve 1: $100 \%$ TEOS; curve 2: TEOS + ibuprofen; curve 3: pure ibuprofen mixed with $\mathrm{KBr}(1: 1$ ratio). The $(2-1)$ trace refers to a differential absorbance spectrum obtained by subtracting curve 1 (100\% TEOS) from curve 2 (TEOS + ibuprofen).
$3850-2500 \mathrm{~cm}^{-1}$ range it is possible to observe some peculiar bands that can be assigned to:

1) The stretching vibration of surface $\mathrm{OH}$ groups at $v \approx$ $3735 \mathrm{~cm}^{-1}$, free from hydrogen bonding interactions.

2) An intense and broad band, located in the 3700$2750 \mathrm{~cm}^{-1}$ range, originating from the stretching vibration of $\mathrm{OH}$ groups mutually interacting by hydrogen bonding.

The activation treatment of the sample influences the hydration state of the surface: in fact, a gradual reduction of water either physisorbed and/or coordinated to the surface of the $\mathrm{SiO}_{2}$ system, characterised by a signal at $v \approx$ $1630 \mathrm{~cm}^{-1}$, due to the in-plane $\mathrm{HOH}$ bending mode (data not reported for the sake of brevity), decreases increasing the evacuation time.

We must consider also the important absorptions present at $v<2100 \mathrm{~cm}^{-1}$. These bands can be attributed, on the basis of their spectral behaviour and of the literature, ${ }^{[21]}$ to Si-O$\mathrm{Si}$ combination (overtones) modes characteristic of the silica matrix. When ibuprofen is added to the pure TEOS system (see curve 2 of Figure 3), some important spectroscopic changes are evident due to the presence of the drug. In particular:

1) At high frequency, in the $2750-3100 \mathrm{~cm}^{-1}$ range, sharp bands appear: their spectral behaviour permits to assign them to $v_{\mathrm{C}-\mathrm{H}}$ stretching modes of all $\mathrm{CH}$-containing species (spectra 2 and 3 in Figure 3) present in the ibuprofen molecule (i.e., of either aliphatic or aromatic nature).

2) At low frequency $\left(v<1800 \mathrm{~cm}^{-1}\right)$ intense and broad bands are observed: these represent either the spectral bending $\left(\delta_{\mathrm{C}-\mathrm{H}}\right)$ counterparts of the above-described stretching modes of all $\mathrm{CH}$-containing species or the $v_{\mathrm{CO}}$ $\left(\approx 1695 \mathrm{~cm}^{-1}\right)$ of the carboxylic residue present in the drug.

As a reference, we reported in Figure 3 the IR spectrum obtained for the pure drug. It is straightforward that there is an almost total overlap between the spectroscopic assignment of the several components which the TEOS +ibuprofen system exhibits and those present in the plain ibuprofen sample. However, all the above components are better visible in the (2-1) trace reported in Figure 3, which represents the difference obtained by subtracting spectrum of plain TEOS from that of the TEOS+ibuprofen system. What is worth noting is the intense negative (i.e., it represents a species that is not present on the system anymore, because it has been "consumed") peak located at $\approx 3740 \mathrm{~cm}^{-1}$ : this experimental evidence is vital in confirming that ibuprofen interacts with the silica surface through the $\mathrm{OH}$ species.

\section{APTES/TEOS samples}

Synthetic procedure optimisation: To increase the drug-surface interactions, but, at the same time, modifying the sur- 
face reactivity (hydrophilic character, wettability, etc.) of the silica, we have prepared an organic-inorganic hybrid matrix by introducing (3-aminopropyl)triethoxysilane (APTES) as precursor in the sol-gel process. Initially we studied a $100 \%$ APTES silica gel and we obtained a monolithic and homogeneous silica/drug carrier. Unfortunately this sample is absolutely unsuitable to sustain and control a real drug delivery. In fact, as expected, it is soluble in aqueous solution and it dissolves completely after few minutes of contact with the physiological medium. As reported by Suratwala ${ }^{[22]}$ the gel-to-sol transition is essentially due to the dissolution of the siloxane cross-links (the break-up of $\mathrm{Si}-\mathrm{O}-\mathrm{Si}$ bonds by hydrolysis during the re-esterification reaction for which the aqueous solution has easy access) that increases with both $\mathrm{pH}$ and temperature of the dissolution medium. High crosslink densities decrease the ability for dissolution and this could be achieved by introducing a second component. To increase the chemical stability of the amino-substituted silica we modified the initial synthesis by using a mixture of TEOS and APTES as starting precursors. The new synthesis is fast and relatively easy relative to that used to synthesise the $100 \%$ TEOS matrix. APTES catalyses the process as an initiating species in the sol-gel reaction: in its absence, hydrolysis and poly-condensation proceed very slowly, lasting weeks instead of a few minutes. The presence of APTES favours the formation of cyclic intermediates, reported in Scheme 2, that probably provides an energetically favoura-

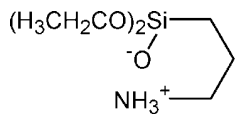

Scheme 2. Cyclic intermediate formed during the hydrolysis/condensation reactions of APTES-TEOS mixture.

ble reaction path. ${ }^{[16]}$ Cyclic intermediates formed during the hydrolysis/condensation reactions of an APTES-TEOS mixture speed up the hydrolysis and allow us to obtain the gel carriers without using acid catalysis. We have prepared samples with increasing APTES amount in the gel composition, as reported in Table 1.

The difference in the reactivity (hydrolysis/condensation) of the two alkoxides (TEOS and APTES; T/A system) has, anyhow, requested a remarkable effort in order to obtain a homogeneous gel. In fact, in the first stage of the study, after water addition to the mixture of the precursors, we obtained a precipitate: after the optimisation of the $\mathrm{H}_{2} \mathrm{O} / \mathrm{alk}$ oxide ratio and the order of addition of reactants (the water

Table 1. Molar ratio of precursors in the samples.

\begin{tabular}{|c|c|c|}
\hline Sample notation $^{[\mathrm{a}]}$ & Volume ratio & Molar ratio of precursors \\
\hline $80 \mathrm{~T} / 20 \mathrm{~A}$ & 1.6:0.4 & $1: 0.22$ \\
\hline $70 \mathrm{~T} / 30 \mathrm{~A}$ & 1.4:0.6 & $1: 0.41$ \\
\hline $60 \mathrm{~T} / 40 \mathrm{~A}$ & $1.2: 0.8$ & $1: 0.58$ \\
\hline $50 \mathrm{~T} / 50 \mathrm{~A}$ & $1: 1$ & 1:0.88 \\
\hline
\end{tabular}

[a] A:APTES; T:TEOS; the ratios are listed in the sequence TEOS/ APTES. is added in the final stage of the process with the opportune amount of drug), we obtained a monolithic and homogeneous gel, requiring only few minutes for its formation. Unfortunately, for the lowest and highest amounts of APTES in the initial mixture (80T/20A and 50T/50A carriers), the final systems appear un-homogeneous and breakable. In these cases the reaction rates of the two alkoxides are not well matched (TEOS is much more resistant to the hydrolysis) and this determines the only partial reticulation of the silica matrix producing the un-homogeneity. This feature is very marked in the tablets derived from the lower percentage of APTES (80T/20A), which are made of white islands of soft consistency with a large amount of supernatant liquid. In the light of this evidence, the $80 \mathrm{~T} / 20 \mathrm{~A}$ sample will not be considered for further investigation. FTIR spectroscopy has been resorted in order to get some information about the surface of the synthesised systems and about the nature of interactions between the ibuprofen molecule and the silica; the results are reported and discussed in the next section.

FTIR spectra of modified systems: The FTIR spectra of the samples, in which the silica matrix has been modified by the presence of APTES, are reported in Figure 4. The OH pat-

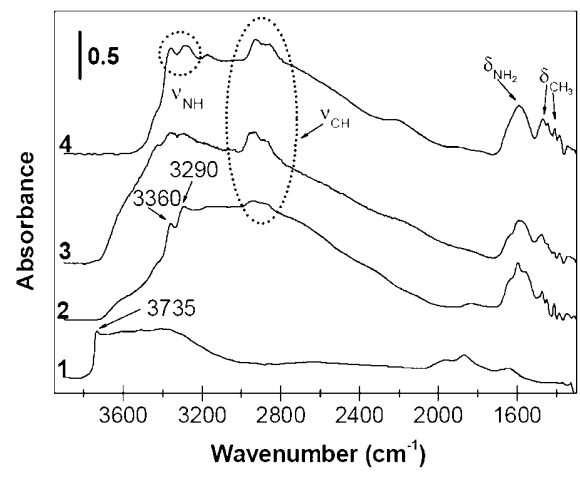

Figure 4. Absorbance FTIR spectra in the $3950-1300 \mathrm{~cm}^{-1}$ range relative to several samples outgassed at beam temperature for one hour: curve 1: $100 \%$ TEOS; curve 2: 70T/30A; curve 3: 50T/50A; curve $4: 100 \%$ APTES.

tern of these systems is very different from that of the pure TEOS material, because of the presence of the APTES compound. In fact, it is possible to observe the absence (of almost all) of the signals generated by the stretching mode due to free $\mathrm{OH}$ (silanols) groups located at about $3745 \mathrm{~cm}^{-1}$ : compare curve 1 to curves 2 and 3 in Figure 3 . In contrast, an intense and broad band, generated by $\mathrm{OH}$ groups interacting through hydrogen bonding, is present in the $3500-2400 \mathrm{~cm}^{-1}$ range. However, some other IR components dominate the spectra of the mixed T/A materials. On the basis of their spectral behaviour and of the literature,$^{[21,23]}$ these bands can be attributed as follows: 
1) In the $3400-3150 \mathrm{~cm}^{-1}$ range the sharp components refer to both symmetric and asymmetric stretching $v_{\mathrm{NH}}$ modes of all surface $\mathrm{NH}$-containing species.

2) At lower frequency, $3100-2750 \mathrm{~cm}^{-1}$, the spectral components are due to the stretching $v_{\mathrm{CH}}$ vibration modes of all $\mathrm{CH}$-containing species.

3) The bands located at $v<1800 \mathrm{~cm}^{-1}$ represent the spectral deformation $\delta$ counterparts of either $\mathrm{NH}$ - or $\mathrm{CH}$-containing species above described.

The new spectral components can be due to the presence of APTES (curve 4 in Figure 4): in fact, this molecule (see Scheme 2) contains several organic groups $\left(-\mathrm{NH}_{2},-\mathrm{CH}_{2}\right.$, $-\mathrm{OC}_{2} \mathrm{H}_{5}$ groups) which can justify the above assignments. Moreover, it is interesting to note that the characteristic silica overtones, located in the $2100-1600 \mathrm{~cm}^{-1}$ range, are totally absent in the mixed 50T/50A system (curve 3 in Figure 4): this might mean that the silica structure is either not preserved during the gelation procedure or the matrix itself failed to form.

When ibuprofen is added to the mixed T/A systems, different characteristics are evident as a function of the T/A ratio: in fact, only in the case of the $70 \mathrm{~T} / 30 \mathrm{~A}$ material can one observe the typical spectral features of the drug besides those of the silica-based system (see curve 1 in Figure 5). Among the several IR components now present, at least three envelopes deserve some attention:

1) The bands indicated by the $v_{\mathrm{NH}}$ in Figure 5 are residual of the APTES presence in the $70 \mathrm{~T} / 30 \mathrm{~A}+$ ibuprofen mixed system, even in the presence of the drug. Under the same conditions no such absorptions are evident in curve 3 (which refers to the $50 \mathrm{~T} / 50 \mathrm{~A}+$ ibuprofen system): only a broad component in the $3700-2900 \mathrm{~cm}^{-1}$ region is observed in this case, most likely due to the $v_{\mathrm{OH}}$ $\mathrm{OH}$ species involved in hydrogen-bonding .

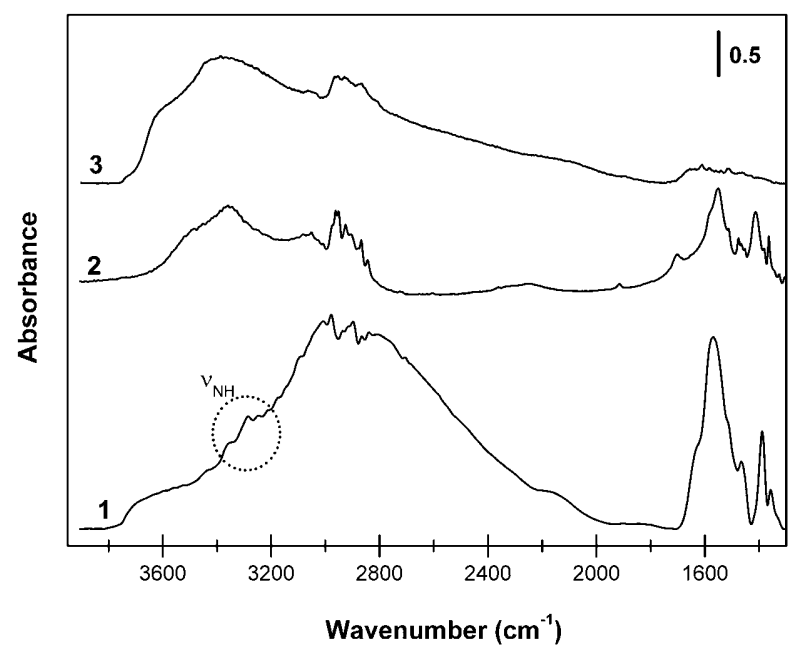

Figure 5. Absorbance FTIR spectra in the $3950-1300 \mathrm{~cm}^{-1}$ range relative to several samples outgassed at beam temperature for one hour: curve 1: 70T/30A + ibuprofen; curve 2: pure ibuprofen in $\mathrm{KBr}$ powder (1:1 ratio); curve $3: 50 \mathrm{~T} / 50 \mathrm{~A}+$ ibuprofen.
2) In the $3100-2700 \mathrm{~cm}^{-1}$ spectral range, a complex absorption is present for both $\mathrm{A} / \mathrm{T}+$ ibuprofen systems: this is due to the $v_{\mathrm{CH}}$ of all $\mathrm{CH}$-containing species present in the materials.

3) At $v<1800 \mathrm{~cm}^{-1}$, only in the case of the $70 \mathrm{~T} / 30 \mathrm{~A}+\mathrm{ibu}-$ profen mixed system, some of the bands typical of either the drug or the APTES modifier are evident. These bands have been described in the above sections and are due to the deformation modes of either $\mathrm{NH}-$ or $\mathrm{CH}-$ containing species.

None of these spectral components are observed for the $50 \mathrm{~A} / 50 \mathrm{~T}+$ ibuprofen materials: this might mean that for this system there is no real interaction between the silica matrix and the drug itself. The FTIR results obtained in the case of the $60 \mathrm{~T} / 40 \mathrm{~A}$ systems are consistent, without significant differences, with that reported for the 70T/30A sample. The drug release behaviour from these carriers will be discussed in the following paragraph.

Drug release: Dissolution tests were carried out in vitro in a simulated body fluid solution at $\mathrm{pH} 7.4$ as reported in the Experimental Section. In the figures the percentage of the drug released is plotted as a function time. The delivery profile from the $50 \mathrm{~T} / 50 \mathrm{~A}$ system is un-controlled (very fast release rate) and absolutely not reproducible (consequently not reported); these are indispensable requisites for its potential application. This is not a surprising result and is certainly related to the physicochemical properties of this carrier. In fact, the absence of a well-defined silica structure and of a real interaction between ibuprofen and the silica surface, as confirmed by FTIR spectroscopy, leads to adverse release behaviour that is not consistent with the aim of our work. The drug-delivery profile from the modified silica carriers $(70 \mathrm{~T} / 30 \mathrm{~A}, 60 \mathrm{~T} / 40 \mathrm{~A})$ is shown in Figure 2, and it is compared with the release curve of the $100 \%$ TEOS carrier. It is worth noting that the results obtained with these systems are absolutely reproducible and, moreover, after 1, 10 and 25 days of ageing the kinetic release is almost unchanged (see Figure 6). This indicates the immediate (after 1 day from their preparation) availability of the samples prepared with the evident advantage derived in the case of a commercial application. We can observe that the presence of APTES in the gel composition has a significant effect in the ibuprofen release leading to a continuous and gradual delivery profile, very similar for the two matrices investigated $(60 \mathrm{~T} / 40 \mathrm{~A} ; 70 \mathrm{~T} / 30 \mathrm{~A})$. This result is more evident if compared with that obtained for the $100 \%$ TEOS derived matrix. As known, the release of biologically active agents from a porous matrix is mainly governed by a combination of several processes, the influence of which on the final release behaviour varies case by case. Among these factors we can name: 1) diffusion through the carrier matrix, 2) carrier matrix erosion, 3) combined erosion/diffusion process and 4) the interaction between matrix and drug molecules. The promising result achieved for our hybrid matrices can be ascribed to a combination of all these factors. Certainly the 


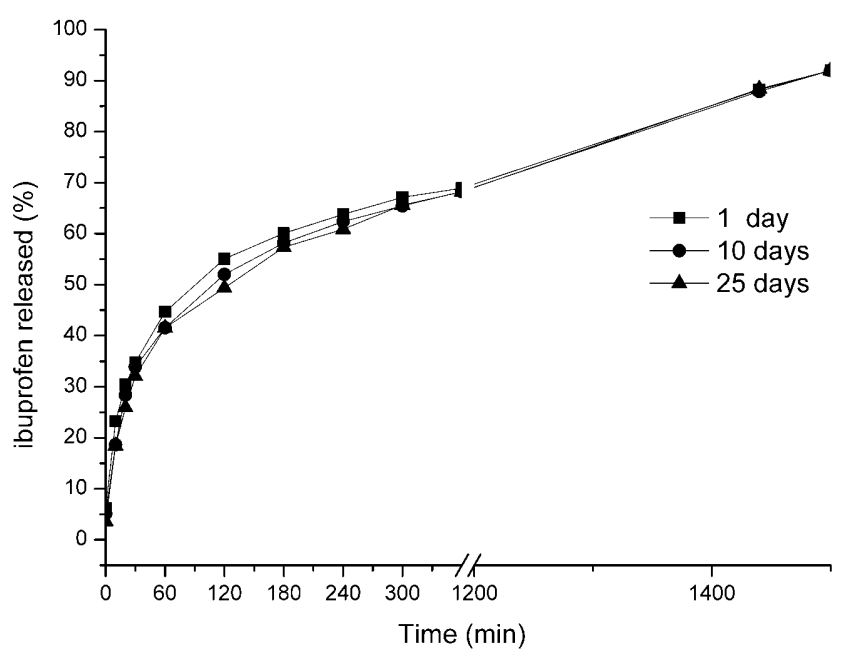

Figure 6. Ageing effect on the ibuprofen release rate.

stronger electrostatic interaction between the carboxy groups in the ibuprofen molecule and the amine groups on the matrices surface (Scheme 3) lead to lower release rates. On the other hand, a strong interaction between the matrix and the drug can be detrimental and can impede the drugrelease process. In fact an essential requisite for a drug-delivery system is to maintain the drug concentration (in vivo) within the range of the therapeutic efficacy and this goal cannot be achieved if only a small fraction of the drug loaded is delivered from the matrix over a relatively long time. In the case of our matrices, the physiological solution, favoured by the increased hydrophilic character, can easily penetrate inside the pores, favouring the dissolution and diffusion of the molecules of drug, surrounded by water; at the same time the major wettability affects the silica dissolution rate in the release medium with a direct influence on the drug-delivery process. It is plausible that the liberation rate is governed by the relationship between the rate of diffusion and dissolution of the siliceous matrix. We can conclude that the delivery profile from the $70 \mathrm{~T} / 30 \mathrm{~A}$ and $60 \mathrm{~T} / 40 \mathrm{~T}$ samples is very similar to that expected from an ideal system (see Figure 2): continuous, gradual and complete (almost all drug loaded has been released).

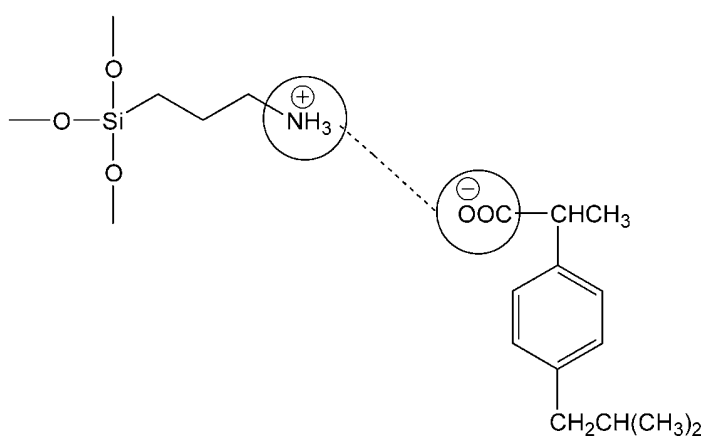

Scheme 3. The interaction between the carboxy groups in the ibuprofen molecule and the amine groups on the silica surface.

\section{Conclusions}

In this study we have developed a reliable and reproducible procedure for to design of a new controlled drug-delivery system. By a one-pot sol-gel process we have prepared silica/ibuprofen tablets functionalised by different amount of aminopropyl groups and we have evaluated the effect of the organic groups amount on the properties of the obtained carriers (physicochemical properties, stability) and on drugdelivery performance. The introduction of an opportune quantity of organic groups allows good control of the hydrophobicity/hydrophilicity ratio and consequently on the drugdelivery process, which is very similar to that expected from an ideal system (gradual and continuous on the time) leading at the same time the release of almost all the drug initially embedded on the carrier. This is a very significant result in the view of an effective commercial application of the studied systems.

\section{Experimental Section}

Materials: Tetraethoxysilane (TEOS; Aldrich), (3-aminopropyl)-triethoxysilane (APTES; Aldrich), $\mathrm{NaOH}$ (Fluka), EtOH (Fluka), ibuprofen sodium salt (Aldrich), Tris buffer saline (Fluka), HCl (Riedel-de Haen). All reagents were used as received.

Synthesis of samples: Drug/silica composites were synthesised by a one step sol-gel process. In a typical procedure the silica alkoxydes (TEOS and/or APTES; $2 \mathrm{~g}$ ) were combined with ethanol and ultra pure water $(0.5 \mathrm{~mL}$ addition of a few drops of $\mathrm{HCl} 0.01 \mathrm{~m}$ in the case of $100 \%$ TEOS matrix) in the required molar ratio (the molar ratio of precursors are reported in Table 1$)$. A solution of ibuprofen sodium salt $(0.2 \mathrm{~g})$ in water $(2 \mathrm{~mL})$ was added to the obtained sol under continuous stirring in order to favour the homogeneous dispersion of the drug in the final material. Each individual sample was obtained by dispensing $1 \mathrm{~mL}$ of the sol into a polyethylene cylindrical vial, the monolithic tablets were left to age in a closed environment for 10 days at room temperature in order to ensure the complete reticulation of the gel and to reach a constant weight $(200 \mathrm{mg}$ ). Each tablet (diameter: $1.0 \mathrm{~cm}$; thickness: $0.5 \mathrm{~cm}$ ) contains $50 \mathrm{mg}$ of ibuprofen.

In vitro release: In vitro desorption of ibuprofen was studied in a saline buffer solution at $\mathrm{pH} 7.4$ (Tris Buffer). In a typical experiment, a silica/ ibuprofen tablet was soaked in the release fluid $(10 \mathrm{~mL})$ maintained at $37^{\circ} \mathrm{C}$. Samples of $1 \mathrm{~mL}$ were removed at predetermined time and replaced by the same volume of the fresh medium. The assays were performed at room temperature with a liquid chromatographic system, Hewlett Packard Series 1100, equipped with an UV detector and with a column LiChrospher 100 RP-18. The mobile phase consisted of $70 \%$ solution $(\mathrm{v} / \mathrm{v})$ of acetonitrile in acidified water; the effluent was monitorated at $270 \mathrm{~nm}$ and the injection volume was $20 \mu \mathrm{L}$. The effective concentration of ibuprofen in solution was calculated on the basis of Equation (1), ${ }^{[1]}$ in which $c_{\text {eff }}$ is the corrected concentration at time $t, c_{\text {app }}$ is the apparent concentration at time $t, v$ is the volume of sample taken and $V$ is the total volume of dissolution medium.

$c_{\text {eff }}=c_{\text {app }}+\frac{v}{V} \sum_{t}^{t-1} c_{\text {app }}$

Each release test was carried out three times by collecting, each time, the data analysis simultaneously from two identical tablets.

Sample characterisation: FTIR spectra were obtained on a BRUKER $113 \mathrm{v}$ spectrophotometer $\left(2 \mathrm{~cm}^{-1}\right.$ resolution, MCT detector). All samples were inspected in the form of self supporting pellets $\left(\approx 15 \mathrm{mg} \mathrm{cm}^{-2}\right)$. All 
samples were activated in controlled atmosphere at beam temperature for one hour in quartz cells connected to a gas vacuum line (residual pressure $<1 \times 10^{-5}$ Torr; 1 Torr $=133.352$ Pascal), in order to get rid of most of the physisorbed species (mainly $\mathrm{H}_{2} \mathrm{O}$ and contaminants weakly interacting with the surface of the several oxidic systems here examined).

[1] E. M. Santos, S. Radin, R. S. Tuan, P. D. Ducheyne, Biomaterials 1999, 20, 1695-1700.

[2] V. Trevisan, M. Signoretto, S. Colonna, V. Pironi, G. Strukul, Angew. Chem. 2004, 116, 4189-4191; Angew. Chem. Int. Ed. 2004, 43, 40974099.

[3] Z. Wu, H. Joo, T. G. Lee, K. Lee, J. Controlled Release 2005, 104, 497-505.

[4] H. Böttcher, P. Slowik, W. Süss, J. Sol-Gel Sci. Technol. 1998, 13, $277-281$

[5] R. Shula, S. Falaize, M. H. Lee, P. Ducheyne, Biomaterials 2002, 23, $3113-3122$.

[6] I. Izquierdo-Barba, A. Martinez, A. L. Doadrio, J. Perez-Pariente, M. Vallet-Regí, Eur. J. Pharm. Sci. 2005, 26, 365-373.

[7] J. Andersson, J. Rosenholm, S. Areva, M. Lindén, Chem. Mater. 2004, 16, 4160-4167.

[8] S.-W. Song, K. Hidajat, S. Kawi, Langmuir 2005, 21, 9568-9575.

[9] P. Horcajada, A. Rámila, G. Férey and M. Vallet-Regí, Solid State Sciences 2006, 8, 1243-1249.

[10] F. Balas, D. Arcos, Angew. Chem. 2007, 119, 7692-7703; Angew. Chem. Int. Ed. 2007, 46, 7548-7558.

[11] K. A. Fisher, K. D. Huddersman, M. J. Taylor, Chem. Eur. J. 2003, 9, 5873-5878.

[12] T. López, P. Quintana, J. M. Martínez, D. Esquivel, J. Non-Cryst. Solids 2007, 353, 987-989.

[13] D. Tebbe, R. Thull, U. Gburek, Acta Biomater. 2007, 3, 829-837.
[14] E. Ghedini, M. Signoretto, F. Pinna, D. Guarascio, G. Cerrato in Studies in Surface Science and Catalysis, Vol.174A (Eds.: A. Gedeon, P. Massiani, F. Babonneau), Elsevier, Amsterdam, 2008, pp. $429-432$.

[15] M. Signoretto, E. Ghedini, F. Pinna, V. Nichele, G. Cerrato in Studies in Surface Science and Catalysis, Vol. 174A (Eds.: A. Gedeon, P. Massiani, F. Babonneau), Elsevier, Amsterdam, 2008, pp. 489-492.

[16] B. V. Zhmud, J. Sonnefeld, J. Non-Cryst. Solids 1996, 195, 16-27.

[17] A. Rámila, B. Muňoz, J. Pérez-Pariente, M. Vallet-Regí, J. Sol-Gel Sci. Technol. 2003, 26, 1199-1202.

[18] F. Qu, G. Zhu, H. Lin, J. Sun, D. Zhang, S. Li, S. Qiu, Eur. J. Inorg. Chem. 2006, 3943.

[19] a) R. V. Albarino, H. Schonhorn, J. Appl. Polym. Sci. 1973, 17, 3323-3335; b) C. L. Bean, Mutat. Res. 1992, 265, 31-44; c) BRRC (1988b), Organofunctional Silane A-1100: In vitro Genotoxicity studies:CHO/HGPRT gene mutation test; Sister Chromatid Exchange Assay; BRRC report number 51-13, 1988, Miller, J. A. (2000) Characterisation of 3-Aminopropyltriethoxysilane (lot No.12013 LU) Technical Report No. 2000-I0000-48641 Dow Corning Corporation Midland, Michigan.

[20] P. Legrand in The Surface Properties of Silicas, Wiley, New York, 1998.

[21] L. J. Bellamy in Advances in Infrared-group Frequencies, Methuen, London, 1968.

[22] T. Suratwala, K. Davidson, Z. Gardlund, D. Collins, D. R. Uhlmann, J. Sol-Gel Sci. Technol. 1998, 13, 553-558.

[23] G. Herzberg in Molecular Spectra and Molecular Structure. II. Infrared and Raman Spectra of Polyatomic Molecules, Van Nostrand, New York, 1945

Received: March 6, 2009

Revised: May 28, 2009

Published online: September 22, 2009 